Crull, K., Weiss, S.

Antibiotic control of tumor-colonizing salmonella enterica serovar Typhimurium

(2011) Experimental Biology and Medicine, 236 (11), pp. 1282-1290 


\title{
Antibiotic control of tumor-colonizing Salmonella enterica serovar Typhimurium
}

\author{
Katja Crull and Siegfried Weiss \\ Molecular Immunology, HZI - Helmholtz Centre for Infection Research, Inhoffenstr 7, D-38124 Braunschweig, Germany \\ Corresponding author: Katja Crull. Email: kko07@helmholtz-hzi.de
}

\begin{abstract}
Systemic administration of Salmonella enterica serovar Typhimurium (S. typhimurium) into tumor-bearing mice results in preferential colonization of tumors and causes shrinkage and sometimes complete tumor clearance. However, in spite of these beneficial antitumor effects, the systemic administration of a bacterial pathogen raises serious safety concerns as well. Addressing those concerns, here, we demonstrate that tumor-colonizing Salmonella can be readily controlled by systemic administration of the antibiotic - ciprofloxacin. Treatment was most effective when started early postinfection. This was achieved at the expense of the efficacy of tumor therapy. In many of the mice treated in such a way, tumors re-grew again. Nevertheless, some mice were able to clear the tumor despite the start of antibiotic treatment only $24 \mathrm{~h}$ after the start of infection. Furthermore, we could demonstrate that such mice had elicited a specific antitumor immune response. Thus, S. typhimurium-mediated tumor therapy might be applied safely when combined with early antibiotic treatment. However, the therapeutic power of the bacteria needs to be enhanced in order to provide a more effective therapeutic tool.
\end{abstract}

Keywords: antibiotics, ciprofloxacin, solid tumor, tumor-targeting, Salmonella typhimurium

Experimental Biology and Medicine 2011; 236: 1282-1290. DOI: 10.1258/ebm.2011.011111

\section{Introduction}

The observation that a bacterial infection might be advantageous for patients with solid cancers was made some time ago. ${ }^{1}$ Although this knowledge exists for more than 130 years and might even date back to ancient Egypt, ${ }^{2}$ the employment of bacteria or bacterial components in tumor therapy has met with little success so far, ${ }^{3}$ due to severe side-effects. However, the extensive acquisition of knowledge and the improvement of techniques in molecular genetics have now rendered the bacterial tumor therapy feasible. Therefore, this approach is presently under intense investigation. ${ }^{4}$

Many pathogenic and non-pathogenic bacterial species have been shown to preferentially colonize solid tumors compared with other healthy tissues, ${ }^{5}$ among them Salmonella enterica serovar Typhimurium (S. typhimurium). ${ }^{4}$ Such bacteria have been shown to cause a strong therapeutic effect on tumors after systemic administration, leading to retardation of tumor growth or even complete clearance. In addition, first clinical trials using highly attenuated S. typhimurium were reported nearly a decade ago. ${ }^{6}$ Nevertheless, the clinical application of Salmonella-mediated tumor therapy is far from being an established therapeutic procedure. However, the tumor invasion process of
S. typhimurium is presently becoming clearer. ${ }^{7}$ Apparently, after intravenous administration, Salmonella enters the tumor via the chaotic vascular system. ${ }^{8-10}$ Proinflammatory cytokines, such as tumor necrosis factor- $\alpha$ (TNF- $\alpha$ ) and interleukin 1, are induced, and disrupt the pathological endothelia of the blood vessels in the tumor. ${ }^{11}$ The developing hemorrhage carries the bacteria into the neoplastic tissue. ${ }^{12}$ Subsequently, the bacteria can thrive in the tumor microenvironment under low oxygen pressure. Thus, protection from phagocytic immune cells and the high nutrient supply provided by dying tumor cells in such an environment result in a longterm colonization of the cancerous tissue. ${ }^{13-15}$

The high cytokine production after systemic application of bacteria can be attributed to various bacterial components as well as virulence factors important for the invasion and infection process. ${ }^{5}$ In particular, the lipopolysaccharides in the cell wall appear to be important in protection. On the one hand it protects the bacteria from phagocytosis, ${ }^{16}$ but on the other hand triggers the complement system and Toll-Like receptor 4 , which in turn activates cytokine production and other host defense systems. ${ }^{17,18}$ Thus, alerting the immune system of the host represents a double-edged sword for Salmonella-mediated tumor therapy. It paves the way for the bacteria into the tumor but represents a 
considerable risk for the patients; ${ }^{19}$ for instance, induction of high levels of TNF- $\alpha$ can lead to septic shock, often with a fatal outcome. ${ }^{20}$

Thus, bacterial pathogens like Salmonella typhimurium need to be attenuated on the one hand to reduce their virulence and on the other hand to reduce the risk of septic shock. The first is usually achieved by metabolic attenuation. There are established bacterial strains that are deficient for certain essential synthesis pathways, like the commonly used $\operatorname{aro}^{-}$mutant that is unable to produce aromatic amino acids ${ }^{21,22}$ or a purI $^{-}$mutant deficient for purine biosynthesis. A systematic survey of the optimally attenuated Salmonella strains has been reported recently. ${ }^{23}$ Similarly, to reduce the risk of septic shock, a strain with an altered lipopolysaccharide has been established. The $m s b B^{-}$mutant strain shows a dramatically reduced induction of TNF- $\alpha$ with human peripheral blood lymphocytes and was well tolerated in clinical trials. ${ }^{24}$ However, reduced TNF- $\alpha$ levels might also reduce the therapeutic potential of the bacteria. ${ }^{4}$

Understandably, alternative ways of bacterial control within the host are needed. In this regard, inducible suicide systems have been considered, ${ }^{25}$ though the most natural alternative appears to be the employment of antibiotics. Salmonella infections are usually self-limiting and only dehydration of the patient has to be avoided. However, in severe cases, fluoroquinolones like ciprofloxacin are the antibiotics of choice. ${ }^{26,27}$ Other antibiotics, commonly used for the treatment of bacterial infections, are used only reluctantly, due to the risk of spreading resistances. ${ }^{28}$

The use of ciprofloxacin to improve the controllability of a bacteria-mediated tumor therapy was already followed in early clinical trials where patients with septic shock symptoms were treated with ciprofloxacin. ${ }^{29}$ However, to our knowledge, no systematic study has been reported investigating the impact of ciprofloxacin treatment on bacteriamediated tumor therapy. Therefore, we have established in our murine CT26 colon carcinoma model, the parameters for ciprofloxacin treatment such as dosing and treatment regimen. As expected, the bacterial infection can be readily controlled by application of ciprofloxacin. Importantly, however, the treatment has to be started early after bacterial application to achieve the optimum results under these circumstances.

\section{Material and methods}

\section{Bacterial strains and growth conditions}

S. typhimurium strain SL7207 (AhisG, $\Delta$ aroA) was kindly provided by Bruce Stocker. ${ }^{21}$ The bacteria were grown on LB (lysogeny broth) agar plates supplemented with $30 \mu \mathrm{g} / \mathrm{mL}$ streptomycin at $37^{\circ} \mathrm{C}$.

\section{Antibiotic treatment in vitro}

A measure of $0.5 \mathrm{~mL}$ of an overnight culture of SL7207 was added to $20 \mathrm{~mL}$ of sterile LB medium, supplemented with $30 \mu \mathrm{g} / \mathrm{mL}$ streptomycin. The culture was incubated at $37^{\circ} \mathrm{C}$ with shaking at $160 \mathrm{rpm}$. Reaching an $\mathrm{OD}_{600}$ of 0.8 , the culture was split and antibiotics were added in the following amounts: kanamycin $(20 \mathrm{mg})$, tetracycline $(2 \mathrm{mg})$ and ciprofloxacin $(2 \mathrm{mg})$. Following the growth curve, samples were taken at distinct time points for turbidity measurement $\left(\mathrm{OD}_{600}\right)$ and plating. Serial dilutions of the cultures were plated on LB agar plates supplemented with $30 \mu \mathrm{g} / \mathrm{mL}$ streptomycin, and colony-forming units (CFU) were counted.

\section{Cell lines and animals}

Female BALB/c mice, at the age of six weeks, were purchased from Janvier (Le Genest-Saint-Isle, France). The CT26 colon carcinoma cell line (CRL-2638; American Type Culture Collection [ATCC], Wesel, Germany) was grown as monolayers in Iscove's Modified Dulbecco's Medium (Gibco BRL [Invitrogen], Karlsruhe, Germany) supplemented with $10 \%(\mathrm{v} / \mathrm{v})$ heat-inactivated fetal calf serum (Gibco BRL [Invitrogen]), $250 \mu \mathrm{mol} / \mathrm{L}$ $\beta$-mercaptoethanol (Serva, Heidelberg, Germany) and 1\% (v/v) penicillin/streptomycin (Sigma, Seelze, Germany).

\section{Infection of tumor-bearing mice and recovery of bacteria from tissue}

Six-week-old female BALB/c mice were subcutaneously injected with $5 \times 10^{5}$ CT26 cells in the abdominal region. When the tumors had reached a size of 5-8 $\mathrm{mm}$ in diameter, mice were injected intravenously with $5 \times 10^{6} \mathrm{CFU}$ of S. typhimurium in phosphate-buffered saline (PBS). Mice were sacrificed at different time points postinfection (pi), and tumor, spleen and liver were removed and weighed. The tissues were then transferred into $1 \mathrm{~mL}$ ( $2 \mathrm{~mL}$ for liver) ice-cold PBS containing 0.1\% (v/v) Triton X-100. The tissue was homogenized with a Polytron PT3000 homogenizer (Brinkmann Kinematica, Lucerne, Switzerland). To determine the CFU per gram of organ, homogenates were serially diluted in PBS and plated on LB agar plates containing streptomycin $(30 \mu \mathrm{g} / \mathrm{mL})$. All animal experiments have been performed with the permission of the local authorities (LAVES) according to the animal welfare act.

\section{Tumor studies}

To follow the development of tumor size, six-week-old female BALB/c mice were injected subcutaneously with $5 \times 10^{5}$ CT26 cells at the abdomen. As soon as small tumors became visible, tumor size was monitored every other day using a caliper. The volume of the tumors was calculated using the formula: $V=4 / 3 \times \pi \times\left(h \times w^{2}\right) / 8$; where $h$ is the height and $w$ is the width. It was assumed that depth and width of the tumor are equal.

\section{Antibiotic treatment in vivo}

Six-week-old female BALB/c mice were subcutaneously injected with $5 \times 10^{5}$ CT26 cells at the abdomen. When the tumors had reached a size of $5-8 \mathrm{~mm}$ in diameter, mice were injected intravenously with $5 \times 10^{6} \mathrm{CFU}$ of S. typhimurium in PBS. Antibiotic treatment was started, on the indicated days after infection. A measure of $100 \mu \mathrm{L}$ 
of antibiotics was administered intraperitoneally every $12 \mathrm{~h}$ on four subsequent days. Control animals were treated with PBS. The health status of the mice was monitored by regular body weight measurement. Mice were humanely euthanized when signs of acute illness became detectable.

\section{Secondary tumors}

After a successful tumor therapy, tumor immunity was tested 100 days after the first tumor injection. CT26 cells, $5 \times 10^{5}$, were injected subcutaneously on the right-hand side of the back, whereas $1 \times 10^{5}$ F1A11 cells were injected under the skin of the left side. Tumor growth was recorded 5 and $10 \mathrm{~d}$ after the secondary injection.

\section{Results \\ Ciprofloxacin is effective against $S$. typhimurium in vitro}

Naturally, S. typhimurium enters the host by the oral route. Ingestion of contaminated food leads to diarrhea but is usually self-limiting. Only severe cases are treated with antibiotics. ${ }^{26,27}$ Therefore, we first wanted to test in vitro, whether the commonly used antibiotics kanamycin, tetracycline and ciprofloxacin allow the control of our S. typhimurium SL7207 strain. Bacterial cultures were grown to an $\mathrm{OD}_{600}$ of 0.1 and split into four samples. Antibiotics were added to three cultures when $\mathrm{OD}_{600} 0.8$ was reached. Upon examination of the turbidity after 1.5, 3 and $20 \mathrm{~h}$, only little differences were observed between the control and the samples treated with kanamycin or tetracycline. In contrast, in samples containing ciprofloxacin, a strongly reduced turbidity was observable even after only $1.5 \mathrm{~h}$ (Figure 1a).

To make sure that this effect is due to bacterial killing, samples were taken at the same time points and plated on selective medium (Figure 1b). The control showed increasing numbers of bacteria which plateaued at five hours. In contrast, reduction of the bacterial numbers was already detectable at $1.5 \mathrm{~h}$ after addition of antibiotics. However, in the sample containing ciprofloxacin, the most rapid decrease in CFU was observed. Only $1 \%$ of bacteria were found to be alive $1.5 \mathrm{~h}$ after initiation of treatment and the titer was 100.000-fold decreased after $20 \mathrm{~h}$. This confirms the superior efficacy of ciprofloxacin for the treatment of S. typhimurium SL7207.

\section{Ciprofloxacin treatment of mice after tumor therapy leads to complete recovery}

Despite the use of attenuated strains, employing S. typhimurium for tumor therapy is accompanied by severe toxicity symptoms of the host. ${ }^{13}$ Besides the elicited immune reaction, the long-term infection is responsible for this. Even when healthy organs like the spleen and liver are colonized to a much lower extent, ${ }^{7}$ the hosts are often moribund and occasionally succumb to the infection. Therefore, attenuation of bacteria aims to achieve a high ratio of tumor colonization compared with healthy organs as well as avoidance of acute toxicity. ${ }^{23}$ However, it is equally important to avoid long-term infections in order
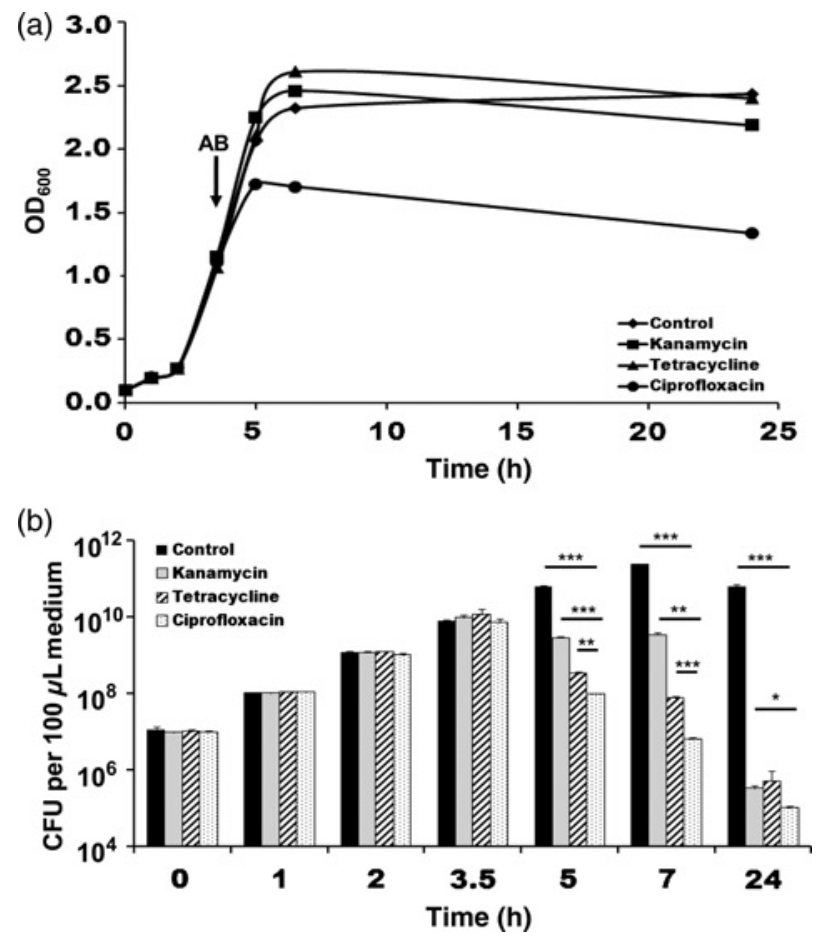

Figure 1 In vitro sensitivity of S. typhimurium SL7207 against kanamycin, tetracycline and ciprofloxacin. (a) Growth curves of SL7207 at $37^{\circ} \mathrm{C}$ in lysogeny broth medium treated with $20 \mathrm{mg}$ kanamycin, $2 \mathrm{mg}$ tetracycline or $20 \mathrm{mg}$ ciprofloxacin. The black arrow indicates the time point of antibiotic (AB) addition. (b) Plating of cultures shown in (a). Bars indicate the mean of three independent samples \pm SDM

to minimize the unwanted side-effects. The latter might also be achieved by antibiotic treatment. Therefore, mice bearing solid CT26 tumors were infected with $5 \times 10^{6}$ SL7207 (day 0). On day 14 pi, antibiotic treatment was started. Body weight was taken as an indicator for the health status of the mice, which was constantly monitored during tumor therapy and the subsequent antibiotic treatment (Figure 2a). All mice showed a comparable drop in body weight directly after infection. The weight remained stable at around $80 \%$ of the initial value. In the control group, receiving PBS, one mouse died on day 17 after infection, whereas the remaining ones survived until the end of the observation period even though their body weight decreased further. Only one mouse recovered (Figure 2a). In the group treated with tetracycline, an intermediate response was observed. One mouse died on day 14 after antibiotic treatment. The surviving mice showed no beneficial response to the antibiotic administration. Similarly, in the kanamycintreated group, two mice died despite the antibiotic treatment, and only one mouse recovered. In contrast, in the group that was treated with ciprofloxacin, all mice survived and recovered completely after initiation of the treatment, as indicated by increasing body weight. Thus, the use of ciprofloxacin is much more effective in controlling the $S$. typhimurium infection than the other two antibiotics.

Eliminating the bacteria at a certain time point after infection might influence their therapeutic potential against the tumor. Thus, we monitored development of the tumor size in the infected mice that were treated with antibiotics or left untreated. Antibiotics were administered on four 
(a)
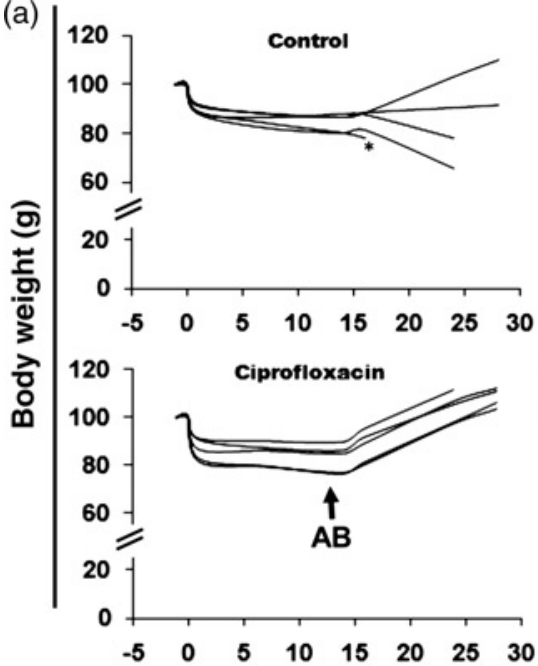

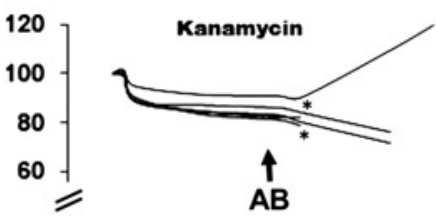

20

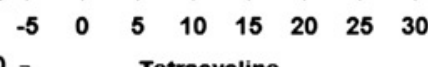

120 Tetracycline

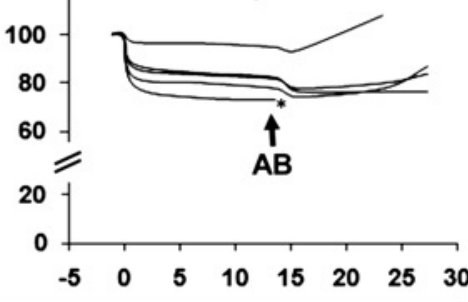

Time (d)

(b)

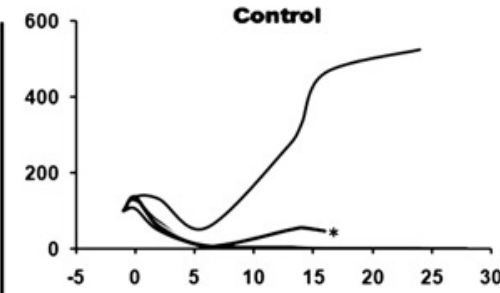

600 Kanamycin
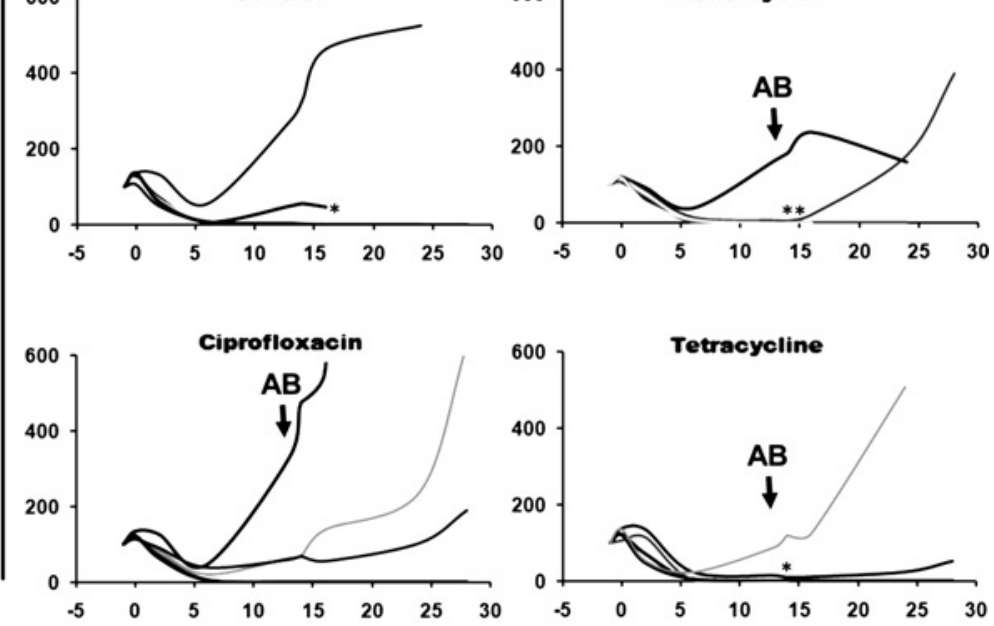
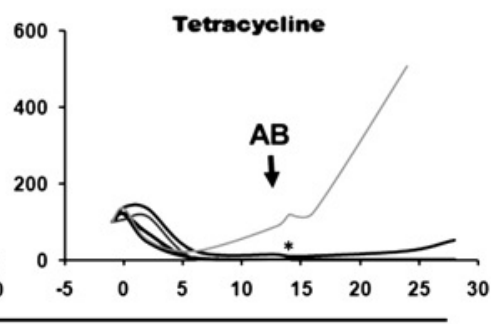

Time (d)

Figure 2 Antibiotic treatment of mice bearing a CT26 tumor infected with S. typhimurium SL7207. Groups of five individual mice are shown. (a) Impact of kana-

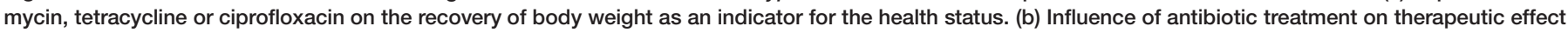
of SL7207 infection. Tumor size is given as a percent of tumor size on day -1 . Black arrows indicate the time point of antibiotic (AB) addition

consecutive days starting at day 14 pi (Figure $2 b)$. Initially, tumor shrinkage could be observed in all groups. In this experimental setting, the tumors in some mice did not respond well to the bacterial therapy and continued to re-grow even before antibiotic treatment. After administration of the antibiotics, the ciprofloxacin-treated mice showed a slightly higher rate of tumor re-growth (Figure $2 b$ ).

To test the hypothesis that a higher rate of tumor re-growth was due to the efficient bacterial killing by ciprofloxacin, the numbers of CFU were measured during the treatment with $2 \mathrm{mg}$ per mouse. Tumor-bearing mice were infected with $5 \times 10^{6}$ SL7207 and ciprofloxacin was given every $12 \mathrm{~h}$ for four consecutive days. Tumor, spleen and liver were removed for plating on days 1, 2, 3, 5 and 15 pi (Figure 3a). The control group, treated with PBS, showed stable bacterial colonization of the liver and only a slight reduction in bacterial numbers was observed in the spleen after $15 \mathrm{~d}$ (Figure 3a). As expected, among all the tissues analyzed, the highest colonization was found in the tumor with $10^{10} \mathrm{CFU}$ per $\mathrm{g}$ one day after infection. This number remained stable until day 2 and then slowly decreased to $10^{7}$ by day 15 . Both treated groups showed comparable colonization on day 1 after infection. However, treatment with 1 as well as $2 \mathrm{mg}$ ciprofloxacin per mouse caused rapid bacterial killing. In all tissues, only $1 \%$ of the initial bacterial colonization persisted by day 2 and bacterial numbers dropped further during the treatment. On day 15 , only single bacterial colonies were recoverable from the different organs examined (Figure 3a).

In parallel, tumor development was measured during S. typhimurium treatment. Compared with the control group which showed complete tumor elimination, both the 1 and $2 \mathrm{mg}$ ciprofloxacin treatment schedules resulted in significant tumor re-growth. During repeated application of $2 \mathrm{mg}$ ciprofloxacin per mouse, tumors in four out of five mice showed strong re-growth after initial shrinkage. 

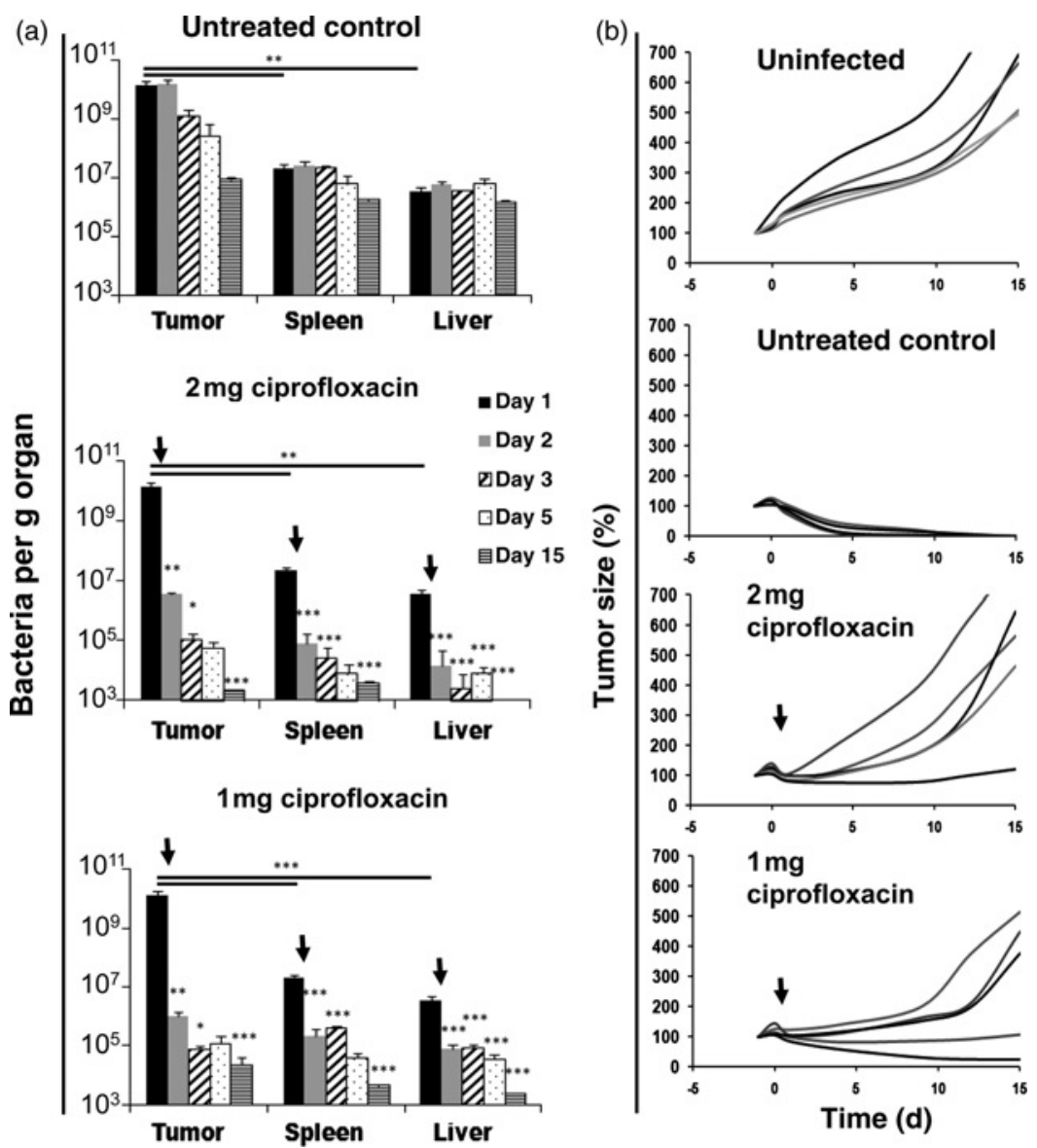

Figure 3 Influence of ciprofloxacin treatment starting $24 \mathrm{~h}$ postinfection (pi) on the efficacy of tumor therapy. (a) Development of bacterial numbers after initiating antibiotic treatment early pi. Bars show colonization of tumor, spleen and liver and represent the mean of five mice \pm SDM. (b) Tumor development during therapy with SL7207 with early initiation of ciprofloxacin treatment. Tumor size of five individual mice per group is given as percent of tumor size on day -1. Black arrows indicate the start of antibiotic treatment

Treatment with $1 \mathrm{mg}$ per mouse showed similar results with re-growth in three out of five tumors and no complete regression. It appears that early ciprofloxacin-based treatment of the bacterial elimination diminishes the antitumor effect of $S$. typhimurium therapy.

\section{Dose-dependent activity of ciprofloxacin in vivo}

We have demonstrated that the effective killing of tumorcolonizing Salmonella by ciprofloxacin early during therapy interferes with the therapeutic effects. Therefore, we examined whether an optimal concentration of ciprofloxacin could be defined in order to control the infection, yet allowing tumor clearance. To study this, treatment with various doses of ciprofloxacin was started at day 7 pi to allow sufficient time for the bacteria to elicit a therapeutic effect (Figure 4a). All animals of the treated groups showed a higher average body weight compared with the control. The best recovery rates were obtained using a dose of 1 or $2 \mathrm{mg}$ ciprofloxacin per mouse for four days. In these groups, all mice recovered completely. Intermediate recovery rates were observed in the groups treated with 0.1 or $0.5 \mathrm{mg}$ per mouse for four days or $1 \mathrm{mg}$ for two days.

Importantly, animals in the intermediate groups receiving $1 \mathrm{mg}$ for two days and $0.1 \mathrm{mg}$ for four days showed a reduced survival rate (Figure $4 \mathrm{~b}$ ). Similarly to animals in the control group, two mice in each group became moribund and had to be killed on days 15 or 16 . In contrast, all animals treated with ciprofloxacin survived the monitored period of the study.

Regression of the tumor size after antibiotic treatment was also investigated (Figure 4c). Representative data from three independent experiments are shown here. A complete tumor clearance in all individuals was observed in the control group. Similar results were obtained for the group treated with $0.1 \mathrm{mg}$ per mouse for four days. Only one outlier was found. Comparatively more mice showed tumor re-growth in the groups treated with other regimens. Two out of five tumors started to re-grow in the groups treated with 0.5 or $1 \mathrm{mg}$ per mouse for four days. After treatment with $1 \mathrm{mg}$ for two days or $2 \mathrm{mg}$ per mouse for four days, three out of five tumors showed massive re-growth. Thus, in the present scenario, optimum bacterial clearance in the mice is achieved at the expense of decreased tumor clearance rates, while optimum tumor clearance leads to severe health problems due to bacterial persistence. This dilemma can be resolved only by the generation of bacteria potentiated with effective tumor clearance capabilities as well as easy controllability. 


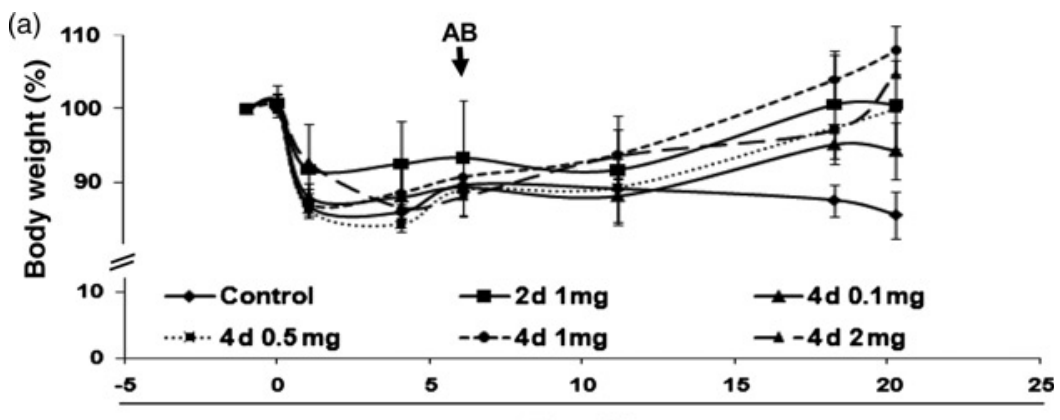

Time (d)
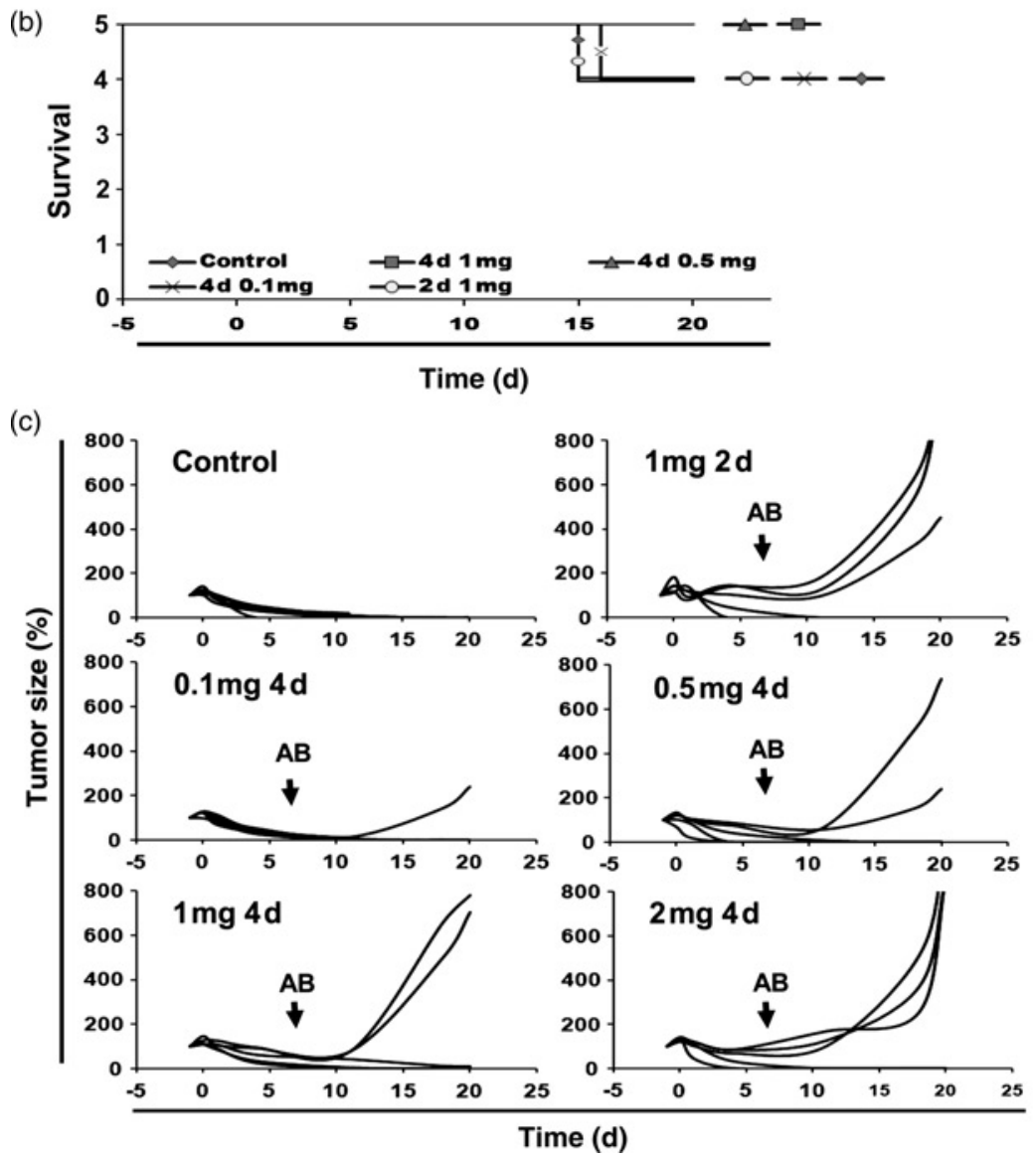

Figure 4 Dose-dependent efficacy of ciprofloxacin to control S. typhimurium SL7207 in tumor-bearing mice. (a) Body weight of mice treated with different amounts of ciprofloxacin. Data represent the mean of five mice \pm SDM (b) Survival of animals is influenced by the amount of ciprofloxacin. Each group represents five mice. (c) Efficacy of tumor therapy exerted by SL7207 is influenced by the amount of ciprofloxacin. Size of tumors is given in percent of tumor size on day -1 for five individual mice. Black arrows indicate the time point of antibiotic (AB) addition

\section{Antibiotic treatment does not impair tumor immunity after treatment}

We had previously noticed that the final clearance of the tumor is most likely due to a T-cell-mediated antitumor response initiated by the bacteria. An important question therefore was, how long the bacteria had to be present in high numbers during tumor therapy to initiate an effective antitumor immunity. To answer this question, ciprofloxacin treatment was started at different time points after infection. Tumor-bearing mice were treated with ciprofloxacin directly after the infection, or 4, 7 and $10 \mathrm{~d} \mathrm{pi}$, and tumor development and survival of the mice were monitored.
Untreated mice started to die by day 10 (Figure 5a). Applying $1 \mathrm{mg}$ ciprofloxacin per mouse at this time point did not bring any effect on the survival of the mice. Similar to the untreated group, only $60 \%$ of the mice survived under these conditions. However, an earlier onset of the antibiotic treatment rescued all of the mice.

Disregarding single outliers, all, except the group in which ciprofloxacin treatment was started immediately after the infection, showed successful clearance of tumors (Figure 5b). Here, only one out of 10 mice showed complete tumor elimination.

Mice that had successfully cleared the tumor were challenged 100 days after initiation of the infection with fresh 

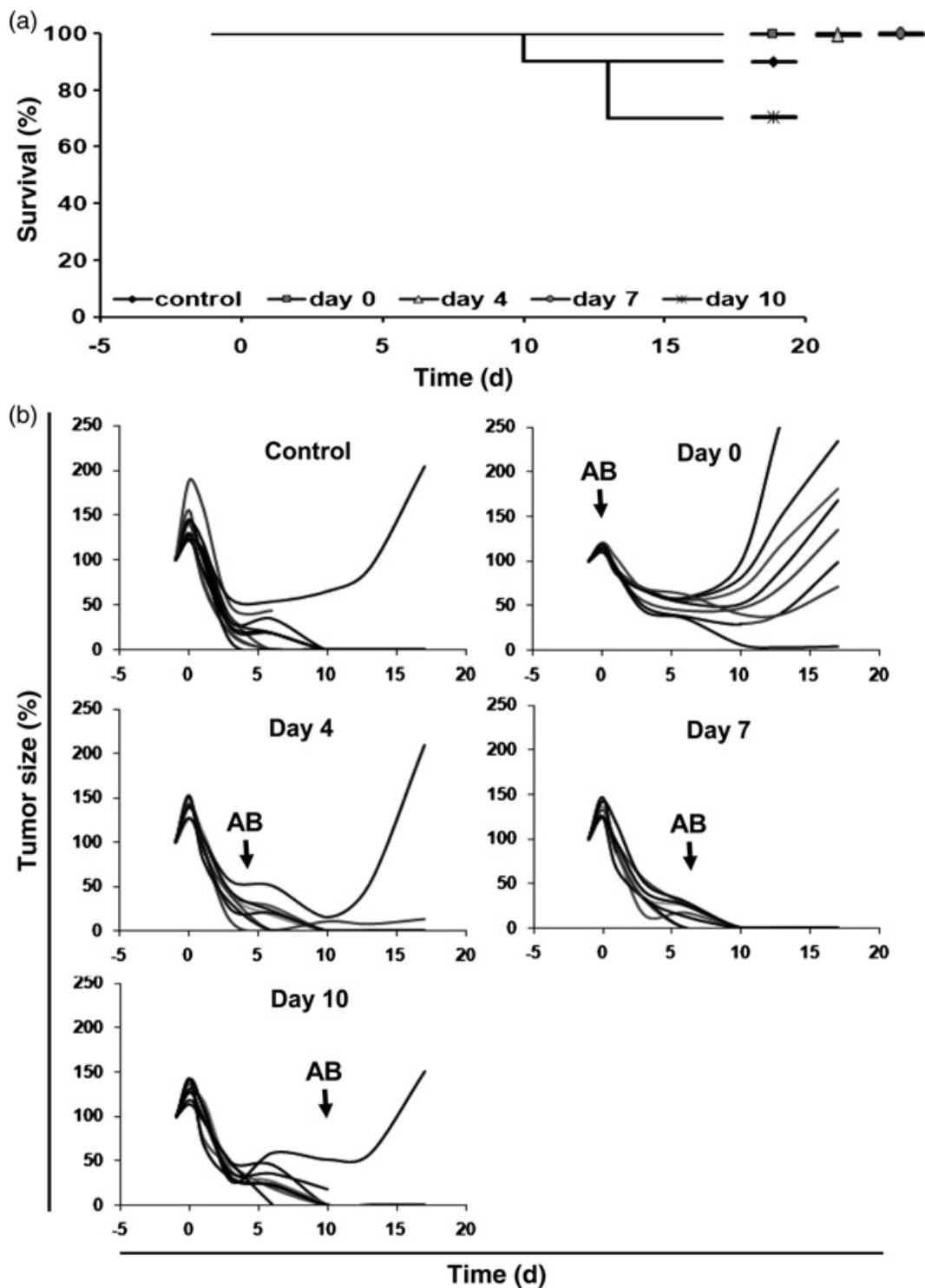

Figure 5 Starting time point of ciprofloxacin treatment influences the efficacy of SL7207-based tumor therapy. (a) Survival of mice. Each group comprised of 10 mice. (b) Efficacy of SL7207-mediated tumor therapy depends on the onset of antibiotic treatment. Tumor size is given in percent of tumor size on day -1 for 10 individual mice. Black arrows indicate the time point of antibiotic (AB) addition

CT26 tumor cells on one flank and with F1A11, a tumor completely unrelated to CT26, on the other flank. All mice independent of the treatment regimen were able to reject the CT26 tumor while the unrelated F1A11 tumor grew in all cases (Table 1). This shows that as long as the primary tumor is cleared after Salmonella administration, an efficient antitumor immunity is induced independent of the antibiotic treatment regimen.

Statistical analysis of Figures 1-5 can be found online at http://ebm.rsmjournals.com/lookup/suppl/doi:10.1258/ ebm.2011.011111/-/DC2 in Supplemental Tables 1-5.

\section{Discussion}

In industrialized countries, cancer is the second most frequent cause of death and the incidence is rising. Thus, the development of novel therapeutic strategies is of utmost importance and one of the most urgent tasks of modern biomedical research. In this regard, bacteria-mediated therapy of solid tumors represents one of such approaches. However, introducing a bacterial pathogen into an often immune-compromised patient is a major challenge to overcome. In this direction, metabolic attenuation of the bacteria is essential and has been achieved for several strains of Salmonella using different essential metabolite synthesis pathways. ${ }^{30}$ However, a recent report has demonstrated that some auxotrophic mutations might interfere with their efficiency of tumor colonization. Thus, safety measures might lower the invasive or therapeutic capacity of the bacteria. ${ }^{23}$ Inducible suicide systems might represent a feasible alternative. Such systems are not available yet and will have to be tested systematically first. For instance, in our hands, employing the inducible lysis gene $E$ of bacteriophage $\Phi \times 174$ reduced the number of the bacteria in the various tissues but resulted in an unacceptable reduction in therapeutic efficacy (Crull, unpublished). 
Table 1 Mice that cleared the primary CT26 tumor after S. typhimurium infection exhibit specific tumor immunity

\begin{tabular}{|c|c|c|c|c|c|c|c|c|c|}
\hline & \multicolumn{4}{|c|}{ Initial tumor challenge } & \multicolumn{5}{|c|}{ Tumor re-challenge } \\
\hline & $\begin{array}{l}\text { CT26 cells } \\
\text { injected }\end{array}$ & $\begin{array}{l}\text { F1A11 cells } \\
\text { injected }\end{array}$ & $\begin{array}{l}\text { Group } \\
\text { size }\end{array}$ & $\begin{array}{l}\text { Complete } \\
\text { responder }\end{array}$ & $\begin{array}{l}\text { CT26 cells } \\
\text { injected }\end{array}$ & $\begin{array}{l}\text { F1A11 cells } \\
\text { injected }\end{array}$ & $\begin{array}{l}\text { Group } \\
\text { size }\end{array}$ & $\begin{array}{l}\text { CT26 } \\
\text { growth }\end{array}$ & $\begin{array}{l}\text { F1A11 } \\
\text { growth }\end{array}$ \\
\hline $\begin{array}{l}\text { Naïve } \\
\text { mice }\end{array}$ & - & - & - & - & $5 \times 10^{5}$ & $1 \times 10^{5}$ & 5 & 5 & 5 \\
\hline Control & $5 \times 10^{5}$ & - & 10 & 8 & $5 \times 10^{5}$ & $1 \times 10^{5}$ & 5 & 0 & 5 \\
\hline Day 0 & $5 \times 10^{5}$ & - & 20 & 3 & $5 \times 10^{5}$ & $1 \times 10^{5}$ & 3 & 0 & 3 \\
\hline Day 4 & $5 \times 10^{5}$ & - & 10 & 8 & $5 \times 10^{5}$ & $1 \times 10^{5}$ & 5 & 0 & 5 \\
\hline
\end{tabular}

The initial CT26 tumor was placed at the abdomen. For tumor re-challenge each tumor was injected at one flank two months after tumor clearance. In the control group, mice were not treated with ciprofloxacin during the first challenge. In the other groups, antibiotic treatment had been started at the indicated day

In the present work, we have systemically investigated the efficacy of antibiotics to control bacterial infection after its therapeutic application. We tested three commonly used antibiotics - kanamycin, tetracycline and ciprofloxacin. Already in vitro, kanamycin and tetracycline showed only low efficacy against S. typhimurium SL7207. Accordingly, no amelioration of the health status could be detected in vivo. Since treatment was started $14 \mathrm{~d}$ pi, the health status of the mice might have been already too low to recover quickly after the relatively small reduction of the bacterial burden. Importantly, because of the additional toxicity, some mice died during the treatment.

However, a four-day schedule administering 1 or $2 \mathrm{mg}$ ciprofloxacin per mouse $14 \mathrm{~d}$ pi resulted in a clear amelioration of the direct effects of $S$. typhimurium infection and greater survival of the tumor-bearing mice. The dose of $2 \mathrm{mg} / \mathrm{d} /$ mouse is just below the $\mathrm{LD}_{50}$ of ciprofloxacin for mice. Accordingly, mice are probably also burdened by the treatment. This might explain the continuous decrease of body weight during the treatment. However, after termination of the treatment, body weight reached the levels observed before tumor therapy. Additionally, only low numbers of bacteria were found in the tissues at that time point. Obviously, the immune system will be sufficient to handle this low number of bacteria as will also be the case when resistant bacteria develop during the treatment. Importantly, $1 \mathrm{mg}$ per mouse had similar effects on bacterial numbers like $2 \mathrm{mg}$, but the toxic burden of the antibiotic was apparently lower.

The efficient removal of the bacterial infection appeared to interfere to some extent with the therapeutic efficiency of the Salmonella therapy. This was even more apparent when ciprofloxacin treatment was started $24 \mathrm{~h}$ after infection. The bacterial infection could be very efficiently controlled with this schedule using either 1 or $2 \mathrm{mg}$ per mouse. However, many of the tumors started to re-grow in this experimental setting, although some of the mice completely cleared the tumors despite the early onset of ciprofloxacin treatment.

As a compromise, in the subsequent experiments, treatment was initialized seven days postinfection. This should allow sufficient time for the therapeutic action of the bacteria but should still allow the effective control of the bacteria. Using different concentrations of ciprofloxacin, we examined the optimal antibiotic treatment regimen. Indeed, when using $0.1 \mathrm{mg}$ ciprofloxacin, all mice cleared the tumor. However, all the mice appeared moribund throughout the observation period. This demonstrates convincingly that with the present genetic makeup of our therapeutic SL7207, it might not be possible to reconcile reasonable bacterial control with the therapeutic maximum. Therefore, bacteria endowed with desired qualities need to be engineered. Several groups have published tumor therapies using recombinant Salmonella that were equipped with prodrug converting enzymes, toxins or antiangiogenic or immune modulatory molecules. ${ }^{4}$ Such bacteria, combined with antibiotic control to avoid long-term infection, might represent the most powerful combination for bacteria-mediated cancer therapy of desirable extent.

After complete clearance of tumors by the Salmonella-mediated therapy, the mice exhibited specific antitumor immunity against the original cancer. No tumor growth could be observed after challenge with the same tumor cells while independent tumor cells grew. In the present case, specific tumor immunity was also observed in mice that had cleared the tumors with additional antibiotic treatment. This held true even when the treatment had started as early as $24 \mathrm{~h}$ after infection. This confirms previous $^{31}$ as well as unpublished data, namely that RAG $2^{-/-}$mice that lack an adaptive immune system are unable to clear the tumors. Thus, the triggering of the specific immune system to elicit an antitumor response in the face of the adjuvant Salmonella could be extremely efficient. This raises the hope that specific immunity can also be generated against other tumors. In addition, immunomodulatory molecules expressed by the bacteria might enhance this effect. Together with antibiotic control, this should represent important steps towards an efficient tumor therapy applicable to human patients.

Author contributions: KC performed experiments, analyzed the data and wrote the manuscript. SW directed the research and revised the manuscript.

\section{ACKNOWLEDGEMENTS}

The authors thank Susanne zur Lage and Regina Lesch for expert technical assistance. This work was supported in part by the Deutsche Krebshilfe, the Ministry of Education and Research (BMBF) and the German Research Council (DFG). 


\section{REFERENCES}

1 Hoption Cann SA, van Netten JP, van Netten C. Dr William Coley and tumour regression: a place in history or in the future. Postgrad Med J 2003;79:672-80

2 Ebbell B. The Papyrus Ebers: The Greatest Egyptian Medical Document. London: Oxford Press, 1937

3 Coley WB. The treatment of malignant tumors by repeated inoculations of erysipelas. With a report of ten original cases. Clin Orthop Relat Res 1893:10:3-11

4 Leschner S, Weiss S. Salmonella-allies in the fight against cancer. J Mol Med 2010;88:763-73

5 Wei MQ, Mengesha A, Good D, Anne J. Bacterial targeted tumour therapy - dawn of a new era. Cancer Lett 2008;259:16-27

6 Low KB, Ittensohn M, Luo X, Zheng LM, King I, Pawelek JM, Bermudes D. Construction of VNP20009: a novel, genetically stable antibiotic-sensitive strain of tumor-targeting Salmonella for parenteral administration in humans. Methods Mol Med 2004;90:47-60

7 Crull K, Bumann D, Weiss S. Influence of infection route and virulence factors on colonization of solid tumors by Salmonella enterica serovar Typhimurium. FEMS Immunol Med Microbiol 2011;62:75-83

8 Graham FO, Coleman PN. Infection of a secondary carcinoma by Salmonella montevideo. Br Med J 1952;1:1116

9 Brown JM, Giaccia AJ. The unique physiology of solid tumors: opportunities (and problems) for cancer therapy. Cancer Res 1998;58:1408-16

10 Harris AL. Hypoxia - a key regulatory factor in tumour growth. Nat Rev Cancer 2002;2:38-47

11 Leschner S, Westphal K, Dietrich N, Viegas N, Jablonska J, Lyszkiewicz M, Lienenklaus S, Falk W, Gekara N, Loessner H, Weiss S. Tumor invasion of Salmonella enterica serovar Typhimurium is accompanied by strong hemorrhage promoted by TNF-alpha. PLoS One 2009;4:e6692

12 Petrache I, Verin AD, Crow MT, Birukova A, Liu F, Garcia JG. Differential effect of MLC kinase in TNF-alpha-induced endothelial cell apoptosis and barrier dysfunction. Am J Physiol Lung Cell Mol Physiol 2001;280:L1168-78

13 Westphal K, Leschner S, Jablonska J, Loessner H, Weiss S. Containment of tumor-colonizing bacteria by host neutrophils. Cancer Res 2008;68:2952-60

14 Avogadri F, Martinoli C, Petrovska L, Chiodoni C, Transidico P, Bronte V, Longhi R, Colombo MP, Dougan G, Rescigno M. Cancer immunotherapy based on killing of Salmonella-infected tumor cells. Cancer Res 2005;65:3920-7

15 Pawelek JM, Low KB, Bermudes D. Tumor-targeted Salmonella as a novel anticancer vector. Cancer Res 1997;57:4537-44

16 Rietschel ET, Kirikae T, Schade FU, Mamat U, Schmidt G, Loppnow H, Ulmer AJ, Zahringer U, Seydel U, Di Padova F. Bacterial endotoxin: molecular relationships of structure to activity and function. FASEB J 1994;8:217-25

17 Gunn JS. The Salmonella PmrAB regulon: lipopolysaccharide modifications, antimicrobial peptide resistance and more. Trends Microbiol 2008;16:284-90
18 Hornef MW, Wick MJ, Rhen M, Normark S. Bacterial strategies for overcoming host innate and adaptive immune responses. Nat Immunol 2002;3:1033-40

19 Guo L, Lim KB, Gunn JS, Bainbridge B, Darveau RP, Hackett M, Miller SI. Regulation of lipid A modifications by Salmonella typhimurium virulence genes phoP-phoQ. Science 1997;276:250-3

20 Bauss F, Droge W, Mannel DN. Tumor necrosis factor mediates endotoxic effects in mice. Infect Immun 1987;55:1622-5

21 Hoiseth SK, Stocker BA. Aromatic-dependent Salmonella typhimurium are non-virulent and effective as live vaccines. Nature 1981;291: 238-9

22 Zhao M, Yang M, Li XM, Jiang P, Baranov E, Li S, Xu M, Penman S, Hoffman RM. Tumor-targeting bacterial therapy with amino acid auxotrophs of GFP-expressing Salmonella typhimurium. Proc Natl Acad Sci USA 2005:102:755-60

23 Arrach N, Cheng P, Zhao M, Santiviago CA, Hoffman RM, McClelland M. High-throughput screening for Salmonella avirulent mutants that retain targeting of solid tumors. Cancer Res 2010;70:2165-70

24 Low KB, Ittensohn M, Le T, Platt J, Sodi S, Amoss M, Ash O, Carmichael E, Chakraborty A, Fischer J, Lin SL, Luo X, Miller SI, Zheng L, King I, Pawelek JM, Bermudes D. Lipid A mutant Salmonella with suppressed virulence and TNFalpha induction retain tumor-targeting in vivo. Nat Biotechnol 1999;17:37-41

25 Loessner H, Endmann A, Leschner S, Westphal K, Rohde M, Miloud T, Hammerling G, Neuhaus K, Weiss S. Remote control of tumour-targeted Salmonella enterica serovar Typhimurium by the use of L-arabinose as inducer of bacterial gene expression in vivo. Cell Microbiol 2007;9:1529-37

26 Denton M. Enterobacteriaceae. Int J Antimicrob Agents 2007;29(Suppl. 3):S9-S22

27 Asperilla MO, Smego RA Jr, Scott LK. Quinolone antibiotics in the treatment of Salmonella infections. Rev Infect Dis 1990;12:873-89

28 Furuya EY, Lowy FD. Antimicrobial-resistant bacteria in the community setting. Nat Rev Microbiol 2006;4:36-45

29 Toso JF, Gill VJ, Hwu P, Marincola FM, Restifo NP, Schwartzentruber DJ, Sherry RM, Topalian SL, Yang JC, Stock F, Freezer LJ, Morton KE, Seipp C, Haworth L, Mavroukakis S, White D, MacDonald S, Mao J, Sznol M, Rosenberg SA. Phase I study of the intravenous administration of attenuated Salmonella typhimurium to patients with metastatic melanoma. J Clin Oncol 2002;20:142-52

30 Zhao M, Yang M, Ma H, Li X, Tan X, Li S, Yang Z, Hoffman RM. Targeted therapy with a Salmonella typhimurium leucine-arginine auxotroph cures orthotopic human breast tumors in nude mice. Cancer Res 2006;66:7647-52

31 Westphal K, Leschner S, Jablonska J, Loessner H, Weiss S. Containment of tumor-colonising bacteria by host neutrophils. Cancer Res 2008;68:2952-60

(Received March 29, 2011, Accepted July 10, 2011) 